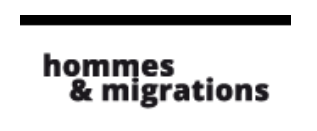

\section{Hommes \& migrations}

Revue française de référence sur les dynamiques

migratoires

1305 | 2014

L'exil chilien en France

\title{
La commémoration des quarante ans du coup d'État du 11 septembre 1973 en Isère
}

\section{Olivier Cogne}

\section{(2) OpenEdition \\ Journals}

\section{Édition électronique}

URL : http://journals.openedition.org/hommesmigrations/2725

DOI : 10.4000/hommesmigrations.2725

ISSN : 2262-3353

\section{Éditeur}

Musée national de l'histoire de l'immigration

\section{Édition imprimée}

Date de publication : 1 janvier 2014

Pagination : $97-103$

ISBN : 978-2919-040261

ISSN : $1142-852 X$

\section{Référence électronique}

Olivier Cogne, «La commémoration des quarante ans du coup d'État du 11 septembre 1973 en Isère », Hommes \& migrations [En ligne], 1305 | 2014, mis en ligne le 01 janvier 2017, consulté le 01 mai 2019. URL : http://journals.openedition.org/hommesmigrations/2725; DOI : 10.4000/ hommesmigrations. 2725 


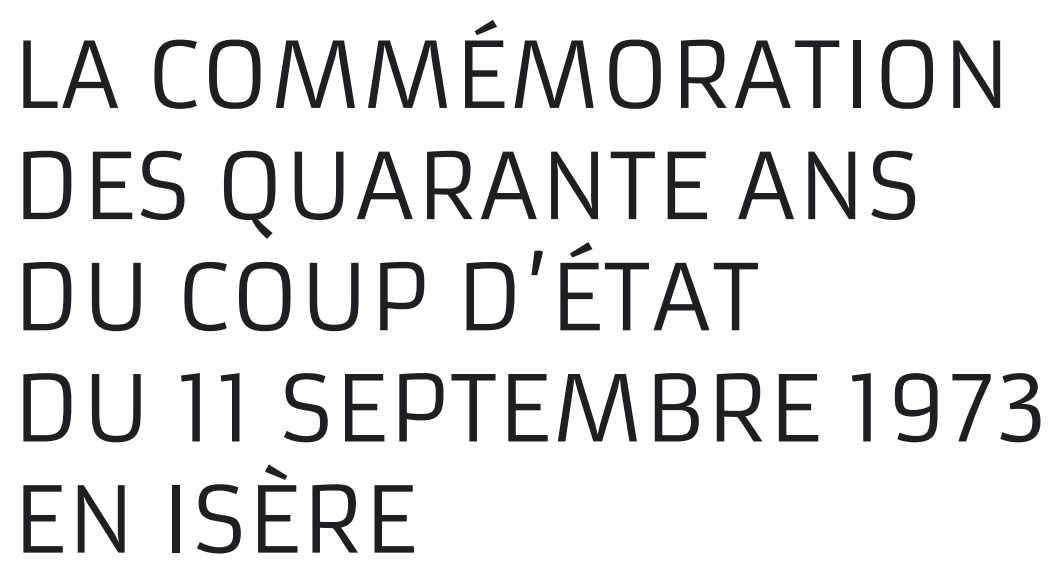

par OLIVIER COGNE, chargé d'expositions au Musée dauphinois, directeur du Musée de la résistance et de la déportation de l'Isère - Maison des droits de l'homme.

\author{
En juin 2013, le Musée de la résistance et de la déportation \\ de l'Isère a présenté à Grenoble une exposition consacrée à \\ I'histoire contemporaine du Chili et à la commémoration \\ du coup d'État de Pinochet. Conçue en synergie avec les acteurs \\ associatifs Iocaux, I'exposition Exiliados. Le refuge chilien en \\ Isère 1973-2013 repose sur une chronologie large, de l'arrivée au \\ pouvoir du président Allende en 1970 à nos jours. La transmission \\ de la mémoire des exilés chiliens qui ont fui la dictature \\ pour s'installer en Isère a fait de cette exposition un succès.
}

La commémoration du coup d'État du 11 septembre 1973 a connu un retentissement notable en Isère. Un tel écho pourrait susciter l'étonnement, car, à l'échelle du territoire national, peu de manifestations pour rappeler la tragédie chilienne se sont déroulées en dehors de la région parisienne. Bien sûr, l'histoire de ce département, qui a été une terre d'accueil pour les exilés chiliens, explique en partie l'impact de cette commémoration, à laquelle les associations locales qui entretiennent des relations avec le Chili et comptent dans leurs rangs d'anciens réfugiés ont pris une part essentielle. Ainsi, le collectif Chili 1973-2013, mémoires et résistances, regroupant une dizaine d'entre elles ${ }^{1}$, voit le jour le 21 février 2013, à Grenoble. Devant l'implication de ses acteurs, les collectivités publiques ont été un soutien efficace sur le plan financier et organisationnel. La ville de Grenoble, qui très tôt s'est 
impliquée dans l'accueil des réfugiés à l'époque de la municipalité socialiste conduite par Hubert Dubedout et le conseil général de l'Isère, a assuré la coordination des initiatives locales. Dès le printemps 2012, le travail de recherche historique engagé par Anouchka Hrdy et celui, derrière la caméra, du réalisateur Michel Szempruch de l'association Repérages autour de la mémoire

Exiliados. Le refuge chilien

en Isère 1973-2013 a été la seule initiative muséale en France qui, à la faveur de la commémoration, répondait à l'objectif de rappeler l'histoire du Chili contemporain et celle des exilés arrivés dans notre pays dans le contexte de la dictature. des Chiliens du département, avaient amorcé le mouvement. C'est dans ce contexte qu'est né le projet d'une exposition au Musée de la résistance et de la déportation de l'Isère, étant donné que les conditions étaient réunies pour développer un projet qui pourrait s'appuyer sur les acteurs associatifs locaux et sur la démarche scientifique et filmique en cours. Inaugurée le 13 juin 2013, cette exposition s'est achevée le 21 octobre suivant. Exiliados. Le refuge chilien en Isère 1973-2013 a été la seule initiative muséale en France qui, à la faveur de la commémoration, répondait à l'objectif de rappeler l'histoire du Chili contemporain et celle des exilés arrivés dans notre pays dans le contexte de la dictature.

\section{Un Musée de la résistance tourné vers les droits de l'homme}

La thématique même entrait pleinement dans le cadre des sujets abordés par le musée qui, s'il est dédié à la connaissance de la Seconde Guerre mondiale, n'en a pas moins vocation à aborder d'autres périodes de l'histoire contemporaine et l'actualité en rapport avec la situation des droits de l'homme dans le monde. L'idée déjà ancienne de pouvoir y aborder un jour le passé récent du Chili s'est trouvée renforcée par l'exposition des photographies de Julio Pantoja (Los Hijos, Tucuman, veinte años después [Les enfants, Tucuman, vingt ans après]) sur les enfants de disparus de la dictature argentine ${ }^{2}$, en 2006, et par le film Terre de refuge ${ }^{3}$ de Favio Fischer et d'Hernán Belón, en 2009, sur l'exil latino-américain dans la région grenobloise. La notion d'exil est, par ailleurs, depuis longtemps au cœur des réflexions du musée, comme en témoignent, par exemple, ces dernières années les travaux expographiques consacrés aux Cambodgiens qui ont quitté leur pays dans le contexte du génocide khmer rouge ${ }^{4}$ ainsi quaux antifascistes italiens arrivés en France après la prise du pouvoir par le Duce 5 . Conforté dans sa démarche par la volonté politique de son institution de tutelle, le conseil général de l'Isère, le musée a alors défini les principaux objectifs de ce travail : contribuer à une meilleure connaissance de l'histoire contemporaine du Chili auprès de la population locale ; établir une première synthèse historique des exilés chiliens en Isère ; mettre en lumière les actions de solidarité dont ils ont bénéficié à l'époque ; créer une synergie avec les acteurs locaux autour de la commémoration ; collecter les témoignages des exilés et de ceux qui les ont aidés.

\section{La muséographie participative à l'œuvre}

Mettant en pratique la démarche qui le caractérise, fondée sur une élaboration véritablement collective du contenu de l'exposition, le musée a réuni en décembre 2012 un groupe de travail au sein duquel ont été invités les associations et les "experts" scientifiques qui avaient travaillé sur ce sujet. Outre la contribution d'Anouchka Hrdy, le musée a pu compter sur la participation de Franck Gaudichaud, maître de conférences en civilisation de l'Amérique

\footnotetext{
2. Dans le contexte d'une autre commémoration, celle des trente ans de l'avènement de la dictature militaire en Argentine. 3. Hernán Belón et Favio Fischer (réal.), Terre de refuge. Récits de l'exil. Tierra de refugio. Historias del exilio, édité par l’AGSPA avec la collaboration du Musée de la résistance et de la déportation de l'Isère - Maison des droits de l'homme, 2009. 4. Jean-Claude Duclos et Olivier Cogne (dir.), Face au génocide, du Cambodge à l'Isère, Grenoble, Musée de la résistance et de la déportation de l'Isère - Maison des droits de l'homme, 2008. 5. Olivier Cogne et Jacques Loiseau (dir.), Libertà ! : antifascistes et résistants italiens en Isère, Grenoble, Musée de la résistance et de la déportation de l'Isère - Maison des droits de l'homme, 2011.
} 


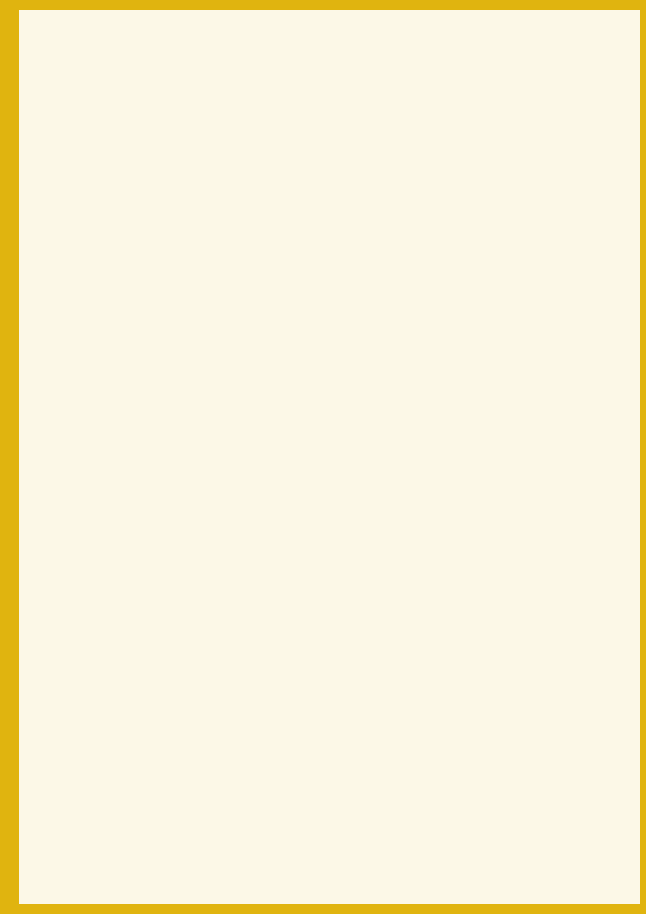

Communication de l'exposition sur la façade du Musée de la résistance et de la déportation à Grenoble (c) Coll. MRDI-MDH

latine à l'université Stendhal (Grenoble-3), auteur d'une thèse récemment publiée sur l'histoire de l'Unité populaire au Chili 6 , de Jacques Barou, chercheur au CNRS, spécialiste à Sciences Po-Grenoble des questions liées aux phénomènes migratoires, et, à distance, sur le concours de Nicolas Prognon, chercheur associé à l'université Toulouse-Le Mirail, et de Fanny Jedlicki, maitre de conférences en sociologie à l'université du Havre, dont les recherches doctorales ont porté sur les refuges chiliens en France et leur retour dans leur pays. Cette réunion a permis de préciser le contenu de l'exposition et du livre $^{7}$ qui devait l'accompagner avec le choix d'un champ chronologique large (de l'arrivée au pouvoir du président Allende en 1970 à aujourd'hui) faisant place à la fois à l'histoire générale et au parcours individuel des Chiliens exilés en Isère. En cela, l'approche retenue ne différait pas de celle du musée habituellement, où le récit local, personnel, coexiste avec un propos plus global. Ces échanges ont mis en évidence la nécessité d'évoquer plusieurs aspects quasi absents du projet initial du musée, comme la place accordée aux Chiliens rentrés dans leur pays natal au lendemain de la dictature, les retornados, bien qu'ils n'aient constitué en Isère qu'une petite minorité. De la même façon, il est apparu essentiel de donner la parole aux jeunes générations, aux Chiliens arrivés enfants ou adolescents en France comme à ceux nés après l'exil, autour des questions d'intégration liées à leur propre vécu et de transmission de celui de leurs parents, dont certains avaient subi la répression de la junte. Le groupe a souligné également qu'il fallait absolument rendre compte de la situation du Chili depuis la fin de la dictature et l'entrée dans l'ère de la "transition démocratique" afin de faire comprendre les difficultés auxquelles le pays est toujours confronté sur le plan politique et juridique.

Par le biais des associations, le musée a entamé un travail de collecte de documents et d'objets auprès des anciens réfugiés demeurés dans le département et de ceux qui les avaient soutenus, et a recueilli parfois leur témoignage. Il a été convenu rapidement avec l'association Repérages de valoriser les entretiens réalisés pour le film en préparation dans l'exposition elle-même sous la forme d'un court montage vidéo. Le musée a effectué, par ailleurs, une recherche dans les archives locales pour compléter ses informations sur l'histoire de l'exil en Isère en dépouillant le fonds du cabinet de la préfecture, qui contient notamment des notes des Renseignements généraux utiles pour établir les statistiques des réfugiés, les actions de soutien des militants locaux et des pouvoirs publics en faveur 
Juan Guzmán aux côtés de Christine Crifo, vice-présidente du Conseil général de l'Isère en charge de la Mémoire et des Droits de l'Homme, parcourt l'exposition Exiliados, le 16 septembre 2013 @ Coll. MRDI-MDH

des Chiliens. Ces investigations ont été complétées par des contacts avec la documentation du quotidien régional Le Dauphiné libéré, qui a relayé ces événements et parfois conservé les photographies qui s'y rapportent. Dans cette quête, les données transmises par l'Office français de protection des réfugiés et apatrides (Ofpra) ont apporté des informations plus précises sur la population chilienne installée en Isère depuis le coup d'État, mais aussi sur ce qu'a été leur trajectoire avant l'exil, avec des précisions sur lâge, la situation familiale et socioprofessionnelle, le lieu de résidence au Chili, les mesures répressives que les exilés ont eu à subir parfois (emprisonnement, assignation à résidence), les pays de transit avant la France, les dates d'arrivée, les raisons de la demande d'asile liées à des activités politiques et syndicales, les rares retours.

\section{Travailler avec le Chili}

En parallèle, considérant qu'un tel travail ne pouvait pas être conduit sans relation avec le pays où les événements s'étaient produits, des contacts ont été engagés avec le Musée de la mémoire et des droits de l'homme de Santiago par l'intermédiaire des acteurs associatifs isérois. Inauguré sous la présidence de Michelle Bachelet en 2010, ce musée tente de lever le voile sur les années de la dictature non sans difficultés dans un pays où le jugement des crimes a été pendant longtemps entravé et continue de l'être et où les programmes scolaires restent silencieux sur la tragédie. Dans un contexte où les héritiers de la junte n'hésitent pas à l'attaquer, son action est d'autant plus remarquable. Saisissant l'opportunité d'une exposition en France, 
Ricardo Brodsky Baudet, son directeur, a apporté son entier soutien à l'initiative iséroise sur le plan historique et documentaire par le prêt de photographies exceptionnelles issues des collections du musée. D’autres liens ont été noués ou développés avec des personnalités réputées pour leur engagement politique et judiciaire au Chili, Manuel Antonio Garretón, professeur de sociologie à l'université catholique de Santiago, et son frère, Roberto Garretón, avocat spécialiste des droits de l'homme ${ }^{8}$, afin de pouvoir bénéficier de leur expertise sur les questions relatives à l'actualité du pays.

\section{Le parcours de l'exposition}

Après plusieurs mois de collecte, le matériau muséographique a été organisé dans l'espace d'exposition suivant les deux niveaux de lecture préétablis (le cadre général de l'histoire du Chili et celui touchant au vécu des (hiliens établis en Isère) au sein de six parties thématiques et chronologiques successives: "Le Chili au temps d'Allende"; "À l'époque de la dictature" ; "L'exil et l'arrivée en France" ; "Refaire sa vie en France"; "Solidarités et résistances"; "Du travail de mémoire et d'histoire". Chacune d'elles a été construite selon la même structuration : un court texte introductif, une dizaine de documents et parfois des objets prêtés par les exilés, accompagnés par une notice. Le musée a fait le choix d'une présentation bilingue de l'ensemble des textes pour une meilleure appropriation de ce travail par les Chiliens et aussi pour intéresser plus largement le public hispanophone vivant ou de passage en Isère et pour constituer un outil pédagogique pour les classes d'espagnol nombreuses dans le département.

Dès l'entrée de l'exposition, le visiteur était confronté à l'atmosphère des années sombres du Chili en ayant face à lui l'une des dernières images connues de Salvador Allende, entouré de sa garde rapprochée, à La Moneda, le jour du coup d'État du 11 septembre 1973. Son dernier discours prononcé dans le palais présidentiel entouré par la junte, retransmis sur les ondes de Radio Magallanes, était diffusé en fond sonore. Invité à traverser cette image, le visiteur se voyait proposer ensuite un retour en arrière historique consacré à l'Unité populaire de 1970 à 1973 et aux espoirs suscités par l'accession au pouvoir de cette coalition des partis de la gauche chilienne avant d'aborder la période de la dictature. Face au mur figurant les portraits de quelques-uns des responsables du régime, des photos de famille sous cadre des Chiliens établis en Isère et emportées au moment du départ témoignaient d'un passé révolu, avant la tragédie.

Le visiteur pénétrait ensuite dans la partie consacrée à l'exil à travers une nouvelle image représentant, à l'aéroport de Santiago, en mars 1976, Cassandra Labrin Donoso et ses quatre enfants qui ont trouvé refuge dans ce département. Quelques objets et photos ont été choisis pour illustrer ce moment charnière de la vie des exilés, parmi lesquels une étiquette de bagage ou un sac remis par le Haut-Commissariat aux réfugiés des Nations unies conservés précieusement jusqu'à aujourd'hui. La suite relatait l'installation en Isère, l'intégration dans la société d'accueil, sans occulter les difficultés liées à l'arrivée dans un nouveau pays et au traumatisme d'un vécu marqué par la répression et l'exil. $\mathrm{Au}$ sein du même espace, un mur était dédié à l'engagement des militants et des pouvoirs publics locaux en
Dès l'entrée de l'exposition, le visiteur était confronté à l'atmosphère des années sombres du Chili en ayant face à lui l'une des dernières images connues de Salvador Allende, entouré de sa garde rapprochée à La Moneda, le jour du coup d'État du 11 septembre 1973. faveur des réfugiés et pour dénoncer la situation à l'époque de la junte sur la base d'affiches, de tracts, de photos de manifestations de rue, de cérémonies organisées dans la région grenobloise. Au terme de cette partie, les témoignages recueillis par l'association Repérages devant la caméra donnaient véritablement corps à ces parcours de vie. 
$\mathrm{Au}$ centre de l'exposition et avant la partie finale, les quinze clichés issus des collections du Musée de la mémoire et des droits de l'homme de Santiago, présentés pour la première fois au Chili au début de l'année 2013 dans l'exposition Fragmentos/Memorias/Imágenes. A 40 años del Golpe (Fragments/Mémoires/Photos. À quarante ans du coup d'État), formaient un espace à part. Prises non sans risque au moment de la dicta-
"Je veux vivre dans un monde où les êtres soient seulement humains, sans autres titres que celui-ci, sans être obsédés par une règle, par un mot, par une étiquette. / Je veux

qu'on puisse entrer dans toutes les églises, dans toutes les imprimeries." Pablo Neruda ture, ces photos témoignent à la fois de la violence de la répression, et des actes de résistance face au pouvoir en place. La scénographie favorisait au maximum l'attention des visiteurs sur ces images d'une rare intensité avec un éclairage précisément cadré sur chacune d'elles dans un espace obscurci. Les légendes du Musée de la mémoire et des droits de l'homme contenaient le minimum d'informations nécessaires à la compréhension de clichés dont la signification est explicite. Reprenant le parcours thématique et chronologique, la dernière partie de l'exposition abordait l'évolution du Chili depuis la fin de la dictature. Le visiteur était invité à franchir une nouvelle image, celle d'une manifestation organisée devant la Chambre des lords à Londres, en décembre 1998, avec un portrait caricaturé d'Augusto Pinochet, alors hospitalisé dans la ville, pour réclamer le jugement de ses crimes. Le dernier espace mêlait d'un côté des documents relatifs au travail de mémoire engagé ces dernières années au Chili par l'érection de monuments commémoratifs, la reconnaissance des principaux lieux de répression dans la capitale, ou l'ouverture du Musée de la mémoire et des droits de l'homme et, de l'autre, des pièces rappelant la tentative avortée de juger l'ancien dictateur, les difficultés de la démocratisation du pays, où la Constitution en vigueur date de la période où la junte était au pouvoir, mais aussi les signes d'espoir suscités par la mobilisation de la jeunesse en 2011 pour que la lumière soit enfin faite sur cette période de l'Histoire et que de véritables réformes politiques, économiques et sociales rompent avec elle. Tel un hommage à Pablo Neruda qui a perdu la vie peu après le coup d'État, le parcours s'achevait sur quelques vers du poète dont la portée touche à l'universel : "Je veux vivre dans un pays où il n'y ait pas d'excommuniés. / Je veux vivre dans un monde où les êtres soient seulement humains, sans autres titres que celui-ci, sans être obsédés par une règle, par un mot, par une étiquette. I Je veux quion puisse entrer dans toutes les églises, dans toutes les imprimeries. / Je veux quion nattende plus jamais personne à la porte d'un hôtel de ville pour l'arrêter, pour l'expulser. / Je veux que tous entrent et sortent en souriant de la mairie. / Je ne veux plus que quiconque fuie en gondole, que quiconque soit poursuivi par des motos. I Je veux que l'immense majorité, la seule majorité: tout le monde, puisse parler, lire, écouter, sépanouir?".

\section{Quels retours du public?}

S'il est difficile d'apprécier la portée d'un tel travail auprès du public, les chiffres de fréquentation ont situé cette exposition parmi les plus visitées du musée avec 3557 personnes, dont une large part d'élèves venus dans le cadre de leur cours d'espagnol. Généralement positifs, les commentaires du livre d'or ont été la plupart du temps laconiques, à quelques exceptions près. Parfois, quelques lignes écrites par des exilés, comme un court témoignage de leur vécu ou pour remercier ceux qui les ont aidés. Le choix même du sujet dans un lieu où la Seconde Guerre mondiale demeure la période de prédilection n'a souffert d'aucune contestation de la part du public. Une personne a même considéré que le musée aurait pu aller plus loin dans sa relation avec le temps présent: "Exposition chilienne très intéressante. Il manque le lien avec la situation politique en France en ce printemps 2013, où des minorités sont agressées par l'extrême droite en toute impunité. Enseigner l'histoire des peuples 
pour éveiller les esprits et comprendre les mécanismes des prises de pouvoir, des origines et des objectifs des guerres et faire de nos jeunes de futurs citoyens lucides et éclairés. 24 juin 2013. J’avais 18 ans en 1976. Je me souviens..."

La mobilisation des acteurs isérois, en premier lieu desquels le collectif Chili 1973-2013, mémoires et résistances, a naturellement contribué à faire connaitre cette exposition, comme la médiatisation par la presse locale (TV, radios, journaux, internet...) a favorisé sa diffusion. Cet écho n'a d'ailleurs pas été spécifique à l'Isère, car sur le plan national, les médias ont accordé une place non négligeable à la commémoration des quarante ans du coup d'État et aux manifestations qu'elle a suscitées.

\section{Plus d'une trentaine d'événements en Isère}

Pendant le temps de l'exposition et au-delà de sa présentation, d'autres manifestations ont été organisées dans le département. Elles ont été la plupart du temps le fait du collectif. Le 11 septembre 2013, quarante ans jour pour jour après le coup d'État, trois cérémonies se sont déroulées dans l'agglomération grenobloise à l'initiative des associations et des collectivités locales : à Saint-Martin-d'Hères, à Pont-de-Claix et à Grenoble. Dans le chef-lieu de l'Isère, quelques centaines de personnes se sont réunies place Salvador-Allende en présence de Jorge Arrate, ancien ministre du Président chilien. En fin de journée, ils étaient aussi nombreux à assister à l'avant-première du film Exilios chilenos de l'association Repérages, organisée en partenariat avec le musée au palais du parlement de Grenoble. Parmi les autres temps forts de ce programme, signalons encore la participation du juge Juan Guzmán (qui a inculpé Augusto Pinochet) à la table ronde "Mémoire et justice : la dictature et le Chili d'aujourd'hui", aux côtés de l'avocate Sophie Thonon, présidente de France Amérique latine, et du journaliste Jac Forton, le 17 septembre suivant. Entre- temps, lors des Journées européennes du patrimoine des 14 et 15 septembre, le musée a proposé au public des visites exceptionnelles de l'exposition, car accompagnées par les exilés qui, pour certains, n'avaient jamais témoigné en dehors du cercle intime. Les échanges ont été marqués par une intense émotion. Enfants ou adolescentes lorsqu'elles ont quitté le Chili ou nées en France après l'exil de leurs parents, quelques personnes appartenant à des générations plus jeunes ont également pris part à ces discussions pour partager leur vécu. En tout, plus d'une trentaine d'activités aux formes les plus diverses (expositions, projections-débats, conférences, colloque, théâtre...) ont été proposées au public entre juin 2013 et janvier 2014.

\section{Et après ?}

L'exposition a sans doute contribué à mieux faire connaître la tragédie chilienne. À l'origine du projet, le musée n'a pas imaginé prolonger ce travail par une version itinérante. En la matière, le livre (voir note 8) qui demeure et le film de Repérages ont vocation bien sûr à être diffusés au-delà des commémorations. De son côté, l'association envisage des suites à son projet filmique en donnant la parole cette fois aux jeunes Isérois d'origine chilienne qui ont exprimé le besoin de pouvoir de leur vécu. Le collectif Chili 1973-2013, mémoires et résistances, qui a rassemblé l'ensemble des acteurs associatifs locaux et permis de fédérer les initiatives, est appelé à disparaître. Il compte néanmoins sur la capacité de ceux qui le composent à se réunir pour œuvrer au travail de mémoire, malgré la diversité des parcours et des idées.

Si une autre actualité a pris place au musée, les relations qui se sont nouées avec les acteurs locaux, mais aussi avec le Chili, devraient aboutir à d'autres projets. À l'heure où ces quelques lignes sont rédigées, un nouveau chapitre de l'histoire de ce pays est sur le point, peut-être, de commencer. 